\title{
Human Papillomavirus E6 Knockdown Restores Adenovirus Mediated-estrogen Response Element Linked p53 Gene Transfer in HeLa Cells
}

\author{
Koji Kajitani ${ }^{1}$, Ken-Ichi Honda ${ }^{2,3 *}$, Hiroyuki Terada ${ }^{2}$, Tomoyo Yasui ${ }^{3}$, Toshiyuki \\ Sumi $^{3}$, Masayasu Koyama ${ }^{3}$, Osamu Ishiko ${ }^{3}$
}

\begin{abstract}
The $p 53$ gene is inactivated by the human papillomavirus (HPV) E6 protein in the majority of cervical cancers. Treatment of HeLa S3 cells with siRNA for HPV E6 permitted adenovirus-mediated transduction of a p53 gene linked to an upstream estrogen response element (ERE). Our previous study in non-siRNA treated HHUA cells, which are derived from an endometrial cancer and express estrogen receptor $\beta$, showed enhancing effects of an upstream ERE on adenovirus-mediated $p 53$ gene transduction. In HeLa S3 cells treated with siRNA for HPV E6, adenovirus-mediated transduction was enhanced by an upstream ERE linked to a $p 53$ gene carrying a proline variant at codon 72, but not for a $p 53$ gene with arginine variant at codon 72 . Expression levels of $p 53$ mRNA and Coxsackie/adenovirus receptor (CAR) mRNA after adenovirus-mediated transfer of an ERE-linked p53 gene (proline variant at codon 72) were higher compared with those after non-ERE-linked $p 53$ gene transfer in siRNA-treated HeLa $S 3$ cells. Western blot analysis showed lower $\beta$-tubulin levels and comparatively higher p53/ $\beta$-tubulin or CAR / $\beta$-tubulin ratios in siRNA-treated HeLa S3 cells after adenovirus-mediated ERE-linked p53 gene (proline variant at codon 72) transfer compared with those in non-siRNA-treated cells. Apoptosis, as measured by annexin $\mathrm{V}$ binding, was higher after adenovirus-mediated ERE-linked $p 53$ gene (proline variant at codon 72) transfer compared with that after non-ERE-linked $p 53$ gene transfer in siRNA-treated cells.
\end{abstract}

Keywords: Adenovirus mediated transduction - codon 72 polymorphism - p53 - siRNA

Asian Pac J Cancer Prev, 16 (18), 8239-8245

\section{Introduction}

p53 is inactivated and degraded by the E6 protein encoded by the integrated human papillomavirus (HPV) in the cervical cancers (Scheffner et al., 1990). The susceptibility of $p 53$ to degradation by the E6 protein varies between the arginine and proline variants at codon 72 of $p 53$ (Storey et al., 1998). In vivo studies demonstrate that the arginine $p 53$ variant is seven times more susceptible to degradation by the E6 protein, compared with the proline variant (Storey et al., 1998). However, the results of clinical studies are controversial, with the majority of studies demonstrating no significant difference between individuals harboring the arginine or proline p53 variant (Andersson et al., 2001; Klug et al., 2009; Nunobiki et al., 2011; Habbous et al., 2012). We considered that hormonal factors may affect these results.

The estrogen response element (ERE) is a DNA sequence (-GGTCAnnnTGACC-) that when bound by the estrogen receptor, leads to the transcriptional activation of target genes (Klinge et al., 2001). We found that the ERE p53 gene with proline at codon 72 promotes adenovirusmediated p53 gene delivery in cultured cells derived from endometrial cancer. This transduction was strong in estrogen receptor-positive HHUA cells and weak in estrogen receptor-negative KLE cells (Kajitani et al., 2012). We studied the effect of the ERE on adenovirusmediated p53 transduction in HeLa S3 cells, which are derived from a human cervical cancer and contain integrated DNA of HPV type 18.

However, the transduction efficiency of a $p 53-$ containing adenovirus vector was low in HeLa S3 cells, even when an ERE was linked to the $p 53$ gene. When E6 mRNA was silenced in HeLa S3 cells by incubation with siRNA for HPV type $18 \mathrm{E} 6$, adenovirus-mediated transduction of the ERE-linked p53 gene (proline variant at codon 72) was restored and early-phase apoptosis rates were increased. These results suggested an unknown effect of ERE on trans-membrane and nuclear transport of the adenovirus-mediated $p 53$ gene (proline variant at codon 72) and therapeutic potential for HPV-associated neoplasm. 


\section{Materials and Methods}

Recombinant adenoviruses containing the human p53 gene

Total RNA was extracted from human chorionic tissue using the RNeasy Mini Kit (Qiagen, Valencia, CA, USA) from a woman who underwent elective abortion at 11 weeks of normal gestation. The woman provided written informed consent for this study, and the study was conducted with the consent of the ethical committee of Osaka City University. cDNAs were synthesized from the total RNA using the High Capacity Reverse Transcription Kit (Applied Biosystems, Foster City, CA, USA). The p53 gene (nucleotides 166-1143, including 21 non-coding nucleotides on the 3' side) was amplified using the following primers; forward primer 5'-GAAGACCCAGGTCCAGAT-3' and reverse primer 5'-TTTATGGCGGGAGGT-3'. To incorporate an EcoRI site together with the ERE and a start codon at the 5' terminus, the following primers were used to amplify the ERE-linked p53 gene; forward primer 5'-GAATTCGGTCATAGTGACCATATGGAAGACC CAGGTCCAGAT-3' and reverse primer 5'-AGTGTGA TGGATATCTGCAGAATTCTTTATGGCGGGAGGT-3'. To incorporate an EcoRI site together with the start codon, but not including the ERE at the 5' terminus, the following primers were used to amplify a non-ERE-linked $p 53$ gene; forward primer 5'-AGGAATTCATGGAAGACCCAGGT C C A G A T - 3, and reverse primer 5'-CAGAATTCTTTATGGCGGGAGGT-3'.

PCR products were agarose gel purified, ligated into the pGEM-T Easy vector (Promega, Madison, WI, USA) and amplified in competent E. coli DH5 $\alpha$ cells (Competent High, Toyobo, Osaka, Japan). For polymorphic variants of p53 at codon 72, the DNA isolated from chorionic tissue encoding the arginine variant (CGC) p53 gene was modified to the proline variant (CCC), using the QuikChange II Site-Directed Mutagenesis Kit (Agilent Technologies, Santa Clara, CA, USA), and the following primers, 5'-GCCAGAGGCTGCTCCCCC-3' and 5'-CGTGCAAGTCACAGACTT-3'. The ERE-linked or non-ERE-linked $p 53$ genes and their codon 72 arginine (EREp53R or p53R) or proline variants (EREp53P or $p 53 \mathrm{P}$ ) were excised by digestion with EcoRV and XhoI, and inserted into the pShuttle-IREShrGFP-1 shuttle vector (Agilent Technologies) (Figure 1). After linearization by Pme I digestion and gel purification without dephosphorylation, the $p 53$ gene constructs were electroporated into BJ5183-AD-1 cells pretransformed with E1-deficient adenovirus vector (Agilent Technologies). The adenoviruses were extracted from the supernatant of AD293 cells after four rounds of rapid freezing in methanol cooled with dry ice and thawing in a $37^{\circ} \mathrm{C}$ water bath and purified using a Virabind Adenovirus Miniprep kit (Cell Biolabs, San Diego, CA, USA) and stored with $10 \%$ glycerol at $-80^{\circ} \mathrm{C}$.

Cell culture and targeted silencing of HPV18 E6 by siRNA

The HHUA human endometrial cancer cell line, which expresses ER $\beta$, was supplied by Riken Laboratories (Wako, Saitama, Japan) (Ishiwata et al., 1984; Zhi et al.,
2007). The KLE human endometrial cancer cell line, which does not express ER $\alpha$ or ER $\beta$, was supplied by DS Pharma Biomedical Co. (Osaka, Japan) ( Kumar et al., 1998). HeLa S3 cells, which express low levels of ER $\alpha$ in our study (data not shown), were a gift from Dr Akira Inoue (Laboratory of Immunology, Osaka City University Graduate School of Medicine, Japan) (Allgood et al., 1992). The presence of the HPV 18 E6 DNA sequence was confirmed in the nuclear DNA of these HeLa cells (data not shown). HeLa S3 cells were cultured in Dulbecco's minimal essential medium supplemented with $10 \%$ fetal bovine serum (Invitrogen, Carlsbad, CA, USA), in a humidified incubator with $5 \% \mathrm{CO}_{2}$ in air. One day prior to treatment of cells with siRNA, cells were detached by treatment with trypsin EDTA, washed, and cultured at $20 \%$ confluence in 24-mm diameter wells of 12-well, plastic tissue culture plates.

For targeted silencing of HPV 18 E6, a 21 bp siRNA hairpin complementary to the HPV 18 E6 mRNA sequence at 755-773 nucleotides (5'-CCACAACGUCACACAAUGU-3') was constructed (Kuner et al., 2007). Cell culture medium was replaced with Opti-MEM medium (Invitrogen) containing the siRNA premixed with Lipofectamine RNAiMAX (Invitrogen) and incubated for $4 \mathrm{~h}$. The medium was subsequently replaced with Dulbecco's minimal essential medium supplemented with $10 \%$ fetal bovine serum, and containing $p 53$ gene-containing recombinant adenoviruses and incubated for $80 \mathrm{~h}$ until cell harvesting. Copy numbers of recombinant adenoviruses were quantified as described below.

Quantification of recombinant adenovirus containing the p53 gene by real-time polymerase chain reaction (PCR)

After cultured cells were lysed with $0.6 \%$ sodium dodecylsulfate (SDS) containing $10 \mathrm{mM}$ EDTA, adenovirus DNA was precipitated with ice-cold ethanol and dissolved in water. For TaqMan real-time PCR of the cell extract or stock

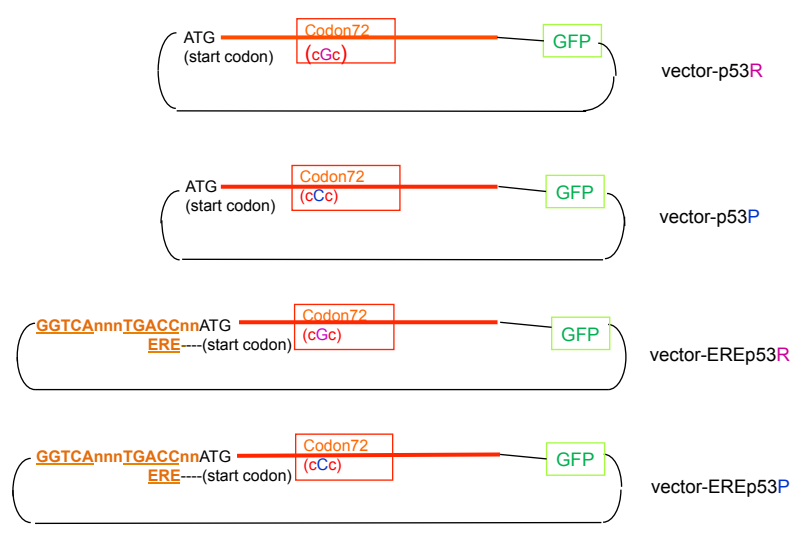

Figure 1. Upstream Estrogen Response Element (ERE)Linked p53 genes with Codon 72 Polymorphisms Inserted into an Adenovirus Vector. Vectors containing arginine variant p53 (p53R), proline variant p53 (p53P), ERElinked p53 with arginine variant (EREp53R), and ERE-linked p53 with proline variant (EREp53P) are shown. A region of the p53 gene (166-1368 nt, indicated by orange lines) was inserted 5 ' to the green fluorescent protein (GFP) gene. The ERE and two additional nucleotides were linked to the 5 ' end of the start codon of $\mathrm{p} 53$ 
HPV E6 Knockdown Restores Adenovirus Mediated-Estrogen Response Element Linked p53 Gene Transfer in HeLa Cells

solution of recombinant adenovirus, the forward primer 5'-GAATTGCTATTATTTGTCGTCATCA-3', reverse primer 5'-AGGTAGACTGACCCTTTTTGGACTT-3', and the probe (CCTTGTAGTCCTCGAGTTA) conjugated with FAM (6-carboxy fluorescein) at the 5' end and the minor groove binder (MGB) at the 3' end (Applied Biosystems), were used for detecting the junction region between the 3' end of the p53 gene insert and the XhoI site of the shuttle vector. The absolute copy number of shuttle vector or recombinant adenovirus was titrated in each well on a 7500 Fast real-time PCR system (Applied Biosystems).

\section{Analysis of p53 mRNA and CAR $m R N A$}

Total RNA was extracted from HeLa S3 cells $80 \mathrm{~h}$ after adenovirus infection, using an RNeasy Mini kit (Qiagen), and cDNA was synthesized using the High Capacity cDNA Reverse Transcription kit (Applied Biosystems). The relative expression of target mRNAs was measured by real-time PCR using TaqMan MGB probes (Hs00153349_m1 for p53, Hs00154661_m1 for CAR), and analyzed in comparison with $18 \mathrm{~S}$ ribosomal RNA, by a multiplex threshold method on a 7500 Fast Real Time PCR System (Applied Biosystems).

\section{Western blot analysis of CAR, p53, and $\beta$-tubulin}

HeLa cells were lysed with $0.1 \%$ SDS $80 \mathrm{~h}$ after adenovirus infection, and the cell extracts were boiled for $2 \mathrm{~min}$ before storage at $-20^{\circ} \mathrm{C}$. The proteins in the cell extracts were treated with DNase I (New England BioLabs, Ipswich, MA, USA), separated by SDS polyacrylamide gel electrophoresis using Miniprotean TGX gel for any kD (BioRad, Hercules, CA, USA) and then transferred to PVDF Hybond P membrane (GE Healthcare). After blocking with 5\% skimmed milk in PBS containing $0.2 \%$ Tween 20 , the membrane was reacted with rabbit antibodies against human CAR (Bethyl Laboratories, Montgomery, TX, USA), human full-length p53 (Santa Cruz Biotechnology, Dallas, TX, USA), or human $\beta$-tubulin (Proteintech, Chicago, IL, USA), washed and then reacted with peroxidase-conjugated anti-rabbit immunoglobulins goat IgG. The chemiluminescent reaction was performed using ECL Prime Western Blotting Detection Reagent (GE Healthcare), and chemiluminescent intensity was quantified using ImageQuant TL software on an LAS4000mini image analyzer (GE Healthcare).

\section{Annexin $V$ binding assays}

HeLa S3 cells were detached $80 \mathrm{~h}$ after adenovirus infection, with $0.25 \%$ trypsin and $1 \mathrm{mM}$ EDTA, washed with PBS, and resuspended in $50 \mathrm{mM}$ Tris- $\mathrm{HCl}$ (pH 8.0), $80 \mathrm{mM} \mathrm{NaCl}, 0.2 \% \mathrm{BSA}$, and $2.5 \mathrm{mM} \mathrm{CaCl} 2$. Cells were subsequently incubated with phycoerythrin-conjugated annexin V (BD Biosciences, San Jose, CA, USA). Annexin $\mathrm{V}$-positive cells were detected by flow cytometry at 564-606 nm, using a FACSCalibur flow cytometer (BD Biosciences).

\section{Statistical analysis}

Data were analyzed by nonparametric methods using PASW statistics 18 Software (SPSS, Chicago, IL, USA), and results with $\mathrm{p}<0.05$ were judged as significant.

\section{Results}

Transduction efficiency of p53-containing adenovirus in HHUA or KLE cells

The efficiency of adenovirus-mediated $p 53$ transduction was lower in KLE cells compared with HHUA cells. The transduction efficiency of ERE-linked $p 53$ was, however, higher than that of non-ERE-linked $p 53$ in either cell line (Figure 2). Although a higher transduction efficiency of ERE-linked p53P compared with ERE-linked p53R was observed in HHUA cells (Figure 2A), no significant difference between the transduction efficiencies of ERElinked $p 53 \mathrm{R}$ and ERE-linked $p 53 \mathrm{P}$ was observed in KLE
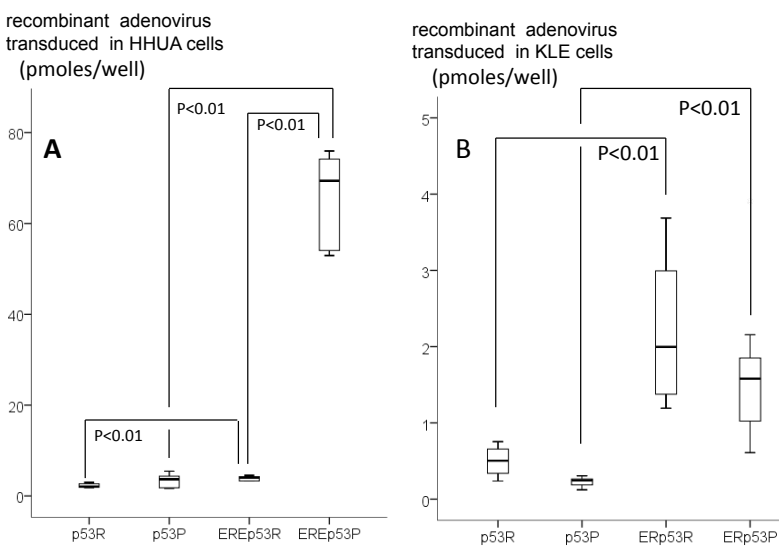

Figure 2. Transduction of p53 genes with Codon 72 Polymorphisms into HHUA and KLE Cells. Copy number of recombinant adenoviruses (p53R, p53P, EREp53R, or EREp53P) in HHUA cells (A), or KLE cells (B) $72 \mathrm{~h}$ after infection. Cells were harvested $72 \mathrm{~h}$ after infection and recombinant adenovirus copy numbers were determined by real time PCR
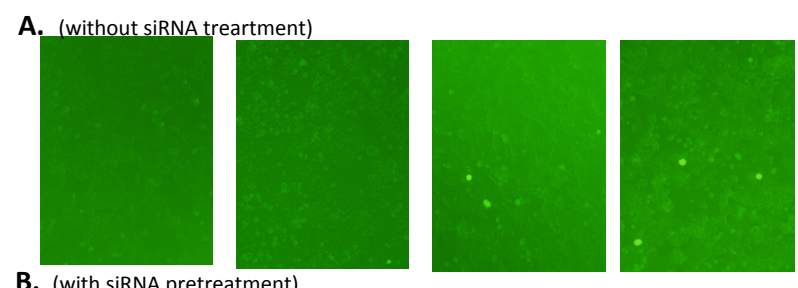

B. (with siRNA pretreatment)

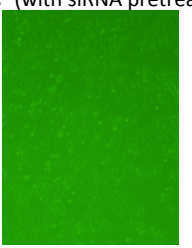

$24 h$

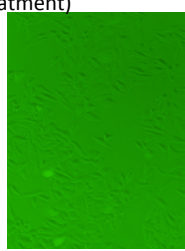

$48 \mathrm{~h}$

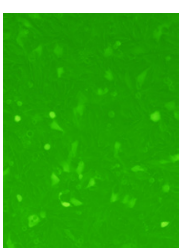

$72 \mathrm{~h}$

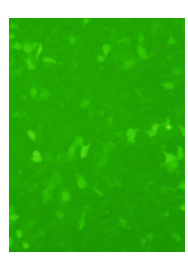

$80 \mathrm{~h}$
Figure 3. Expression of GFP in HeLa S3 cells following transduction with $p 53$ P recombinant adenovirus. GFP was assessed by fluorescence microscopy $24,48,72$, and $80 \mathrm{~h}$ following infection of HeLa S3 cells with ERE linked-p53P adenovirus without (A) or with (B) pretreatment with HPV 18 E6 siRNA. GFP expressed in the cells was visualized with an emission wavelength of $488 \mathrm{~nm}$ and photographed with a $530 / 40 \mathrm{~nm}$ filter on an inverted fluorescence microscope (IX71; Olympus, Tokyo, Japan) 


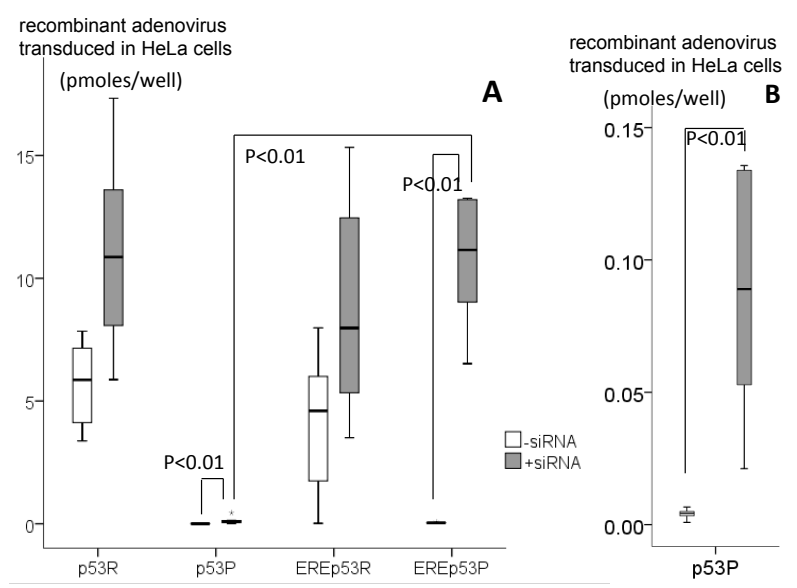

Figure 4. Transduction Efficiency of the p53 gene in HeLa S3 Cells. HeLa S3 cells, pretreated with HPV 18 E6 siRNA (gray column) or no siRNA (white column), were infected with p53R, p53P, EREp53R, or EREp53P gene-containing adenovirus. Adenovirus copy number was determined by real-time PCR, $80 \mathrm{~h}$ after infection. The results of $\mathrm{p} 53 \mathrm{P}$ are magnified in $\mathrm{B}$

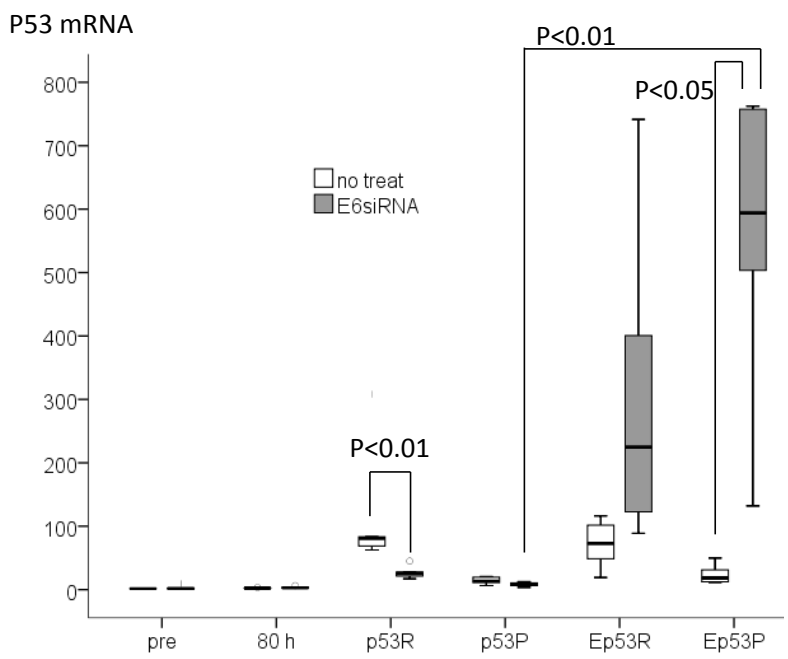

Figure 5.p53 mRNA Levels in HeLa S3 Cells Following Infection with $p 53$ gene-containing Adenoviruses. HeLa S3 cells pretreated with HPV 18 E6 siRNA (gray column) or no siRNA (white column), were infected with p53R, p53P, EREp53R, or EREp53P gene-containing adenovirus and cells were harvested after $80 \mathrm{~h}$. Relative levels of p53 mRNA were analyzed by real-time PCR

cells (Figure 2B).

Transduction efficiency of p53-containing adenovirus in HeLa S3 cells following pretreatment with HPV18 E6 siRNA

When HeLa S3 cells were pretreated with HPV 18 E6 siRNA, the number of cells positive for $p 53$-containing adenovirus (as measured by the percentage of GFPpositive cells) was increased markedly $72 \mathrm{~h}$ after infection with ERE-linked $p 53 \mathrm{P}$-containing adenovirus (Figure 3B). When HeLa S3 cells were not pretreated with HPV18 E6 siRNA, the number of cells positive for $p 53$-containing adenovirus remained low $72 \mathrm{~h}$ after infection with ERElinked $p 53 \mathrm{P}$-containing adenovirus (Figure $3 \mathrm{~A}$ ).

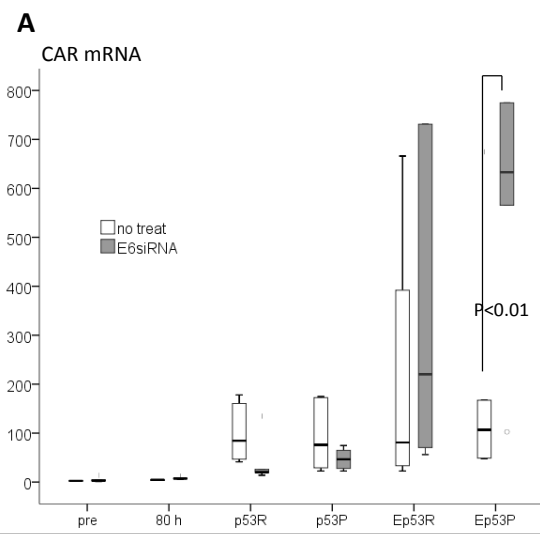

B

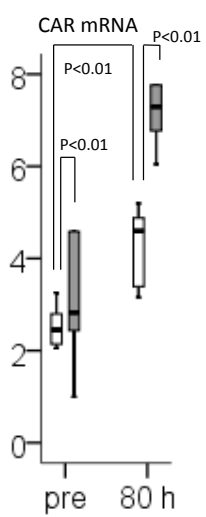

Figure 6. Levels of Coxsackie and Adenovirus Receptor (CAR) mRNA in HeLa S3 Cells Following Infection with p53 gene-containing Adenoviruses. HeLa S3 cells pretreated with HPV 18 E6 siRNA (gray column) or no siRNA (white column) were infected with p53R, p53P, EREp53R, or EREp53P adenoviruses and cells were harvested after $80 \mathrm{~h}$. Relative levels of CAR mRNA were analyzed by real-time PCR. The results of pre-infection and $80 \mathrm{~h}$ after infection with adenovirus vector are magnified in $\mathrm{B}$

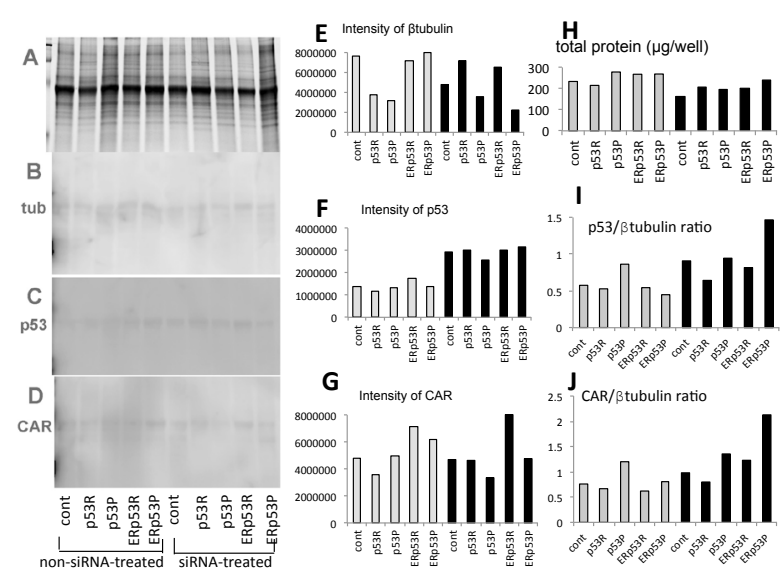

Figure 7. SDS Polyacrylamide Gel Electrophoresis of Proteins in HeLa Cell Extracts and Western Blot Analysis for $\boldsymbol{\beta}$-tubulin, CAR, and p53. HeLa S3 cells were pretreated with HPV 18 E6 siRNA (black column) or without siRNA (gray column) and incubated for $80 \mathrm{~h}$ after infection of p53R, p53P, EREp53R(ERp53R), or EREp53P(ERp53P) gene-containing adenoviruses or no adenovirus (cont). Proteins in these cells were separated by SDS polyacrylamide gel electrophoresis, and stained with Coomassie brilliant blue R (A), or analyzed by western blotting with anti- $\beta$ - tubulin (B), anti-p53 (C), anti-CAR (D) IgG. Chemiluminescent intensity of CAR, p53, and $\beta$-tubulin band areas were measured by subtracting near-background intensity, and $\mathrm{p} 53 / \beta$-tubulin (I) and CAR/ $\beta$ tubulin $(\mathrm{J})$ ratios were calculated

We observed no significant difference in the efficiency of HeLa S3 cell transduction between p53R-containing adenovirus and upstream ERE-linked p53R-containing adenovirus. Pretreatment of HeLa S3 cells with siRNA for HPV18 E6 significantly increased the transduction of ERE-linked p53P-containing adenovirus (Figure 4A) and non-ERE-linked $p 53 \mathrm{P}$-containing adenovirus (Figure 4B). Furthermore, the transduction efficiency of ERE- 
annexin $\mathrm{V}$

binding cell rates

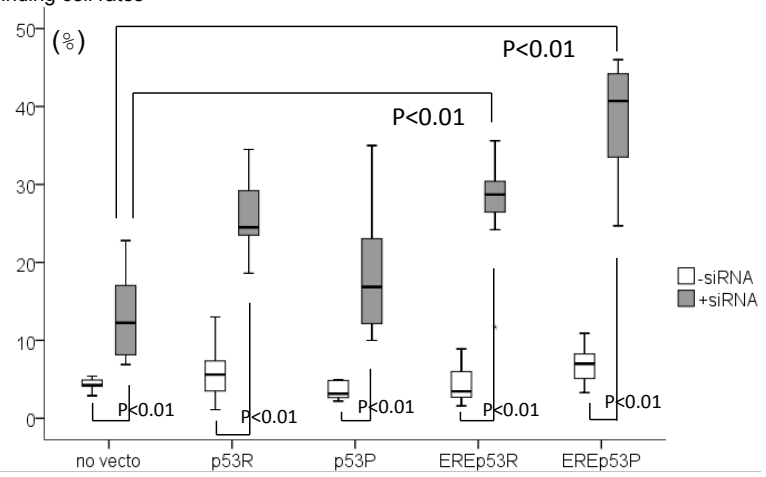

Figure 8. Analysis of Annexin V Binding in HeLa S3 Cells Following Transduction of p53 gene-containing Adenoviruses. HeLa S3 cells pretreated with HPV 18 E6 siRNA (gray column) or no siRNA (white column), were infected with p53R, p53P, EREp53R, or EREp53P gene-containing adenoviruses or no adenovirus (no vecto), and $80 \mathrm{~h}$ later annexin $\mathrm{V}$ binding was assessed by flow cytometry

linked $p 53 \mathrm{P}$ adenovirus was significantly higher than that of non-ERE-linked p53P adenovirus (Figure 4A). However, pretreatment of HeLa S3 cells with siRNA for HPV18 E6 did not change the transduction efficiency of ERE-linked p53R-containing adenovirus, and there was no difference between the transduction of ERE-linked p53R-containing adenovirus and non-ERE-linked p53Rcontaining adenovirus (Figure 4A).

Analysis of p53 mRNA and CAR mRNA levels in HeLa $S 3$ cells following transduction with p53-containing adenovirus

p53 mRNA levels were higher in siRNA-treated cells compared with non-siRNA-treated cells $80 \mathrm{~h}$ after infection with ERE-linked $p 53 \mathrm{P}$-containing adenovirus (Figure 5). Furthermore, $p 53$ mRNAlevels in HeLa S3 cells pretreated with HPV18 E6 siRNA were higher in cells infected with ERE-linked $p 53 \mathrm{P}$-containing adenovirus compared with non-ERE-linked p53P-containing adenovirus (Figure 5). However, p53 mRNA levels were not higher in siRNAtreated cells compared with non-siRNA-treated cells $80 \mathrm{~h}$ after infection with ERE-linked or non-ERE-linked p53Rcontaining adenovirus (Figure 5). Levels of CAR mRNA were significantly higher in siRNA-treated cells compared with non-siRNA-treated cells, $80 \mathrm{~h}$ after infection with ERE-linked p53P-containing adenovirus. CAR mRNA levels were also higher in HeLa S3 cells infected with ERE-linked $p 53 \mathrm{P}$ adenovirus compared with non-ERElinked $p 53 \mathrm{P}$ adenovirus (Figure 6A). CAR mRNA levels in HeLa S3 cells uninfected with either ERE-linked or non-ERE-linked $p 53$-containing adenovirus, were higher in cells treated with HPV18 E6 siRNA compared with cells not treated with HPV18 E6 siRNA (Figure 6B).

Western blot analysis and quantification of $C A R / \beta$-tubulin and $p 53 / \beta$-tubulin ratios

The chemiluminescent intensity of western blot bands corresponding to $p 53, \mathrm{CAR}$, and $\beta$-tubulin (Figure B-D) was analyzed and is shown graphically in Figure 7E-G. Total protein content in each sample was slightly lower in siRNA-treated HeLa cells compared with non-siRNAtreated cells (Figure $7 \mathrm{H}$ ), and $\beta$-tubulin content was lowest in siRNA-treated HeLa cells $80 \mathrm{~h}$ after infection with ERE-linked $p 53 \mathrm{P}$-containing adenovirus (Figure 7E). The $p 53 / \beta$-tubulin and CAR/ $\beta$-tubulin ratios were largest in siRNA-treated HeLa cells $80 \mathrm{~h}$ after infection with ERElinked $p 53 \mathrm{P}$-containing adenovirus (Figure 7I-J).

\section{Flow cytometric analysis of annexin $V$ binding rates in HeLa S3 cells}

Annexin $\mathrm{V}$ binding rates were significantly higher in siRNA-treated HeLa S3 cells compared with untreated HeLa S3 cells, even when cells were not exposed to $p 53-$ containing adenovirus (Figure 8, no vector). Annexin $\mathrm{V}$ binding rates were also significantly higher in siRNAtreated HeLa S3 cells infected with ERE-linked p53containing adenovirus compared with cells not infected with p53-containing adenovirus (Figure 8). However, annexin $\mathrm{V}$ binding was not significantly higher in siRNAtreated HeLa S3 cells infected with non-ERE-linked p53containing adenovirus compared with cells not infected with $p 53$-containing adenovirus.

\section{Discussion}

siRNA targeting the HPV oncogene E6 and/or E7 up-regulates expression of $p 53$ and $\mathrm{Rb}$ in head and neck cancer cell lines (Adhim et al., 2013). Furthermore, co-expression of E6-specific siRNA and wild-type p53 suppresses the growth of human cervical cancer lines $(\mathrm{Li}$ et al., 2013). These findings indicate that intrinsic p53 expression is suppressed by HPV E6 and that knockdown of E6 is essential for $p 53$ expression. However, additional treatments were required to induce apoptosis of cervical cancer cells (Hougardy et al., 2006; Tan et al., 2012).

In this study, treatment of HeLa cells with siRNA for HPV E6 permitted adenovirus-mediated transfer of the p53 gene (proline variant at codon 72). However, the transduction rate was large only when the $p 53$ gene contained proline at codon 72 and was linked to an upstream ERE. These phenomena suggest unknown promoting effects of the ERE on adenovirus-mediated p53 gene transfer.

Expression of p53 and ER $\alpha$ is associated with the induction of leukemia inhibitory factor (LIF) during fertilized egg implantation into the endometrium and higher LIF induction was observed in mice transgenic for the human $p 53 \mathrm{R}$ gene compared with mice transgenic for the human $p 53$ P gene (Feng et al., 2011). A common set of genes has also been shown to be more strongly transcribed by $p 53 \mathrm{R}$ compared with $p 53 \mathrm{P}$ (Jeong et al., 2010). In our study, however, estrogen receptors binding with the ERE may work as a transcription factor and trigger expression of other genes involved in the transduction of $p 53 \mathrm{P}$.

The proline-rich domain of $p 53$ (encoded by codons 64-93) is required for transcriptional control of target genes (Liu et al., 2003; Bergamaschi et al., 2006). We constructed a p53P-containing adenovirus vector containing this domain and produced a high level of 
transduction of the $p 53 \mathrm{P}$ gene when linked to an upstream ERE in estrogen receptor-expressing HHUA cells. We also constructed ERE- and non-ERE-linked p53R genes, but found no significant difference between their efficiency of transduction in HHUA cells. Similar results were obtained in estrogen receptor-expressing SW48 cells, which are derived from colon cancer (Drewinko et al., 1984; Kajitani et al., 2012). Low transduction efficiency was observed in non-ER-expressing KLE cells, with relatively higher transduction rates for ERE-linked p53P and ERE-linked p53R gene-containing adenovirus, compared with nonERE-linked $p 53$ gene-containing adenovirus. However, no effect of upstream ERE-linkage was observed in HeLa S3 cells, and pretreatment of cells with HPV E6 siRNA restored the promoting effect of the upstream ERE for p53P transduction, but not for $p 53 \mathrm{R}$ transduction.

Pretreatment of HeLa S3 cells with siRNA for HPV type 18 E6 increased the efficiency of transduction of p53P, suggesting that the inhibitory effects of HPV E6 on adenovirus-mediated $p 53$ gene delivery in these cells target the $p 53$ gene with proline at codon 72 . Furthermore, the effect of pretreatment of HeLa S3 cells with siRNA for HPV type 18 E6 was limited to transduction of ERElinked $p 53 \mathrm{P}$.

Restoration of adenovirus-mediated $p 53$ gene delivery to HeLa cells by HPV E6 siRNA suggests inhibitory effects of HPV E6 on the cellular responses to ERErelated gene expression. Increased levels of $p 53$ and CAR mRNAs suggest that transcription of these genes is also ERE related.

The p53 codon 72 polymorphism appears to affect the transduction of the $p 53$ gene; with transduction being more efficient for $p 53 \mathrm{P}$ than for $p 53 \mathrm{R}$. These phenomena indicate that differences in the molecular structure of $p 53$, which is synthesized from adenovirus-mediated transfer of the p53 gene, affect cellular responses.

p53 is rapidly degraded in normal cellular process but is stabilized in response to DNA damage, microtubule disruption, or other intracellular events. p53 is also stabilized by some oncoproteins (Blagosklonny MV., 1997). It is considered that siRNA treatment of the HPV E6 oncogene increased accumulation of $p 53$ protein after adenovirus-mediated transfer of the $p 53$ gene.

We constructed an adenovirus vector containing the human chorionic gonadotropin $\beta$ chain gene (HCG $\beta$ ) to study whether the effect of an upstream ERE on the transduction efficiency of linked genes is specific for $p 53$, but we did not observe any difference in the transduction efficiency of ERE- and non-ERE-linked HCG $\beta$ adenovirus in HHUA cells (data not shown). These results suggest that the transferred $p 53$-specific cellular response occurs against subsequently transferred exogenous $p 53$ genes. These phenomena are, at least in part, dependent on transcriptional changes, including the induction of CAR mRNA.

Under cellular stress, the $p 53$ gene is activated and leads to cell-cycle arrest, DNA repair, and/or apoptosis, via associated protein complexes, which control the transcription of target genes (Trigiante et al., 2006; Kruse et al., 2009). The ERE binds estrogen receptors in addition to other transcriptional factors, and may lead to activation of downstream target genes. Menendez et al. (2010) showed that an ERE-conjugated p53 response element could synergistically transactivate $p 53$ target genes (Menendez et al., 2010). The effect of an upstream ERE on the transcription of p53 and CAR mRNA seems to depend on transcriptional complexes; however, p53 gene transduction may also be affected by other factors, as shown by the significantly higher transduction rates of adenovirus-mediated p53P in siRNA-treated cells compared with non-siRNA-treated cells.

There are many benefits of adenovirus-mediated $p 53$ gene therapy for the treatment of human cancer. However, low rates of $p 53$ transduction have limited the extent of these applications (Chen et al., 2014). We expect that transfer of adenovirus-mediated ERE-linked $p 53$ into cancer cells or dysplastic cells of the cervix following pretreatment with HPV E6 siRNA may lead to apoptosis of these HPV integrating cells.

\section{Acknowledgements}

We thank Tetsuo Shoji MD, Takuya Misugi MD, and Tomoko Wakasa MD for supporting this study.

\section{References}

Adhim Z, Otsuki N, Kitamoto J, et al (2013). Gene silencing with siRNA targeting E6/E7 as a therapeutic intervention against head and neck cancer-containing HPV16 cell lines. Acta Oto-Laryngologica, 133, 761-71.

Allgood VE, Cidlowski JA (1992). Vitamine B6 modulates transcriptional activation by multiple members of the steroid hormone receptor superfamily. J Biol Chem, 267, 3819-24.

Andersson S, Rylander E, Strand A, et al (2001). The significance of p53codon 72 polymorphism for the development of cervical adenocarcinomas. Br J Cancer, 85, 1153-6.

Bergamaschi D, Samuels Y, Sullivan A, et al (2006). iASPP preferentially binds $p 53$ proline-rich region and modulates apoptotic function of codon 72-polymorphic p53. Nat Genet, 38, 1133-41.

Blagosklonny MV (1997). Loss of function and p53 protein stabilization. Oncogene, 15, 1889-93.

Chen G, Zhang S, He X, et al (2014). Clinical utility of recombinant adenoviral human $p 53$ gene therapy: current perspectives. OncoTargets Therapy, 7, 1901-9.

Drewinko B, Yang LY, Leibovitz A, et al (1984). Cellular discriminants for a biological classification of human colon carcinoma. Cancer Res, 44, 4241-53.

Feng Z, Zhang C, Kang H-J, et al (2011). Regulation of female reproduction by $p 53$ and its family members. FASEB J, 25, 2245-55.

Habbous S, Pang V, Eng L, et al (2012). p53Arg72Pro polymorphism, HPV status and initiation progression and development of cervical cancer: A systematic review and meta-analysis. Clin Cancer Res, 18, 6407-15.

Hougardy BMT, Maduro JH, van der Zee ADJ, et al (2006). Proteasome inhibitor MG132 sensitizes HPV-positive human cervical cancer cells to rhTRAIL-induced apoptosis. Int $J$ Cancer, 118, 1892-900.

Ishiwata I, Ishiwata C, Soma M, et al (1984). Establish01ment of human endometrial adenocarcinoma cell line containing estradiol-17beta and progesterone receptors. Gynecol Oncol, 17, 281-90. 
Jeong BS, Hu W, Belyi V, et al (2010). Differential levels of transcription of p53-regulated genes by the arginine/ proline polymorphism: $p 53$ with arginine at codon 72 favors apoptosis. FASEB J, 24, 1347-53.

Kajitani K, Honda K, Terada H, et al (2012). Estrogen response element enhances adenovirus-mediated transfer of the $p 53$ gene according to codon 72 polymorphisms and cellular estrogen receptor expression. J Cancer Ther Res, 31, 1-8.

Klinge CM (2001). Estrogen receptor interaction with estrogen response elements. Nucleic Acids Res, 29, 2905-19.

Klug SJ, Ressing M, Koenig J, et al (2009). TP53 codon 72 polymorphism and cervical cancer: a pooled analysis of individual data from 49 studies. Lancet Oncol, 10, 772-84.

Kumar NS, Richer J, Owen G, et al (1998). Selective downregulation of progesterone receptor isoform $\mathrm{B}$ in poorly differentiated human endometrial cancer cells: Implications for unopposed estrogen action. Cancer Res, 58, 1860-5.

Kuner R, Vogt M, Sultmann H, et al (2007). Identification of cellular targets for the human papillomavirus E6 and E7 oncogenes by RNA interference and transcriptome analyses. $J$ Mol Med, 85, 1253-62.

Kruse JP, Gu W (2009). Modes of p53 regulation. Cell, 137, 609-22.

Li X, Li Y, Hu Z, et al (2013). Plasmid-based E6-specific siRNA and co-expressioin of wild type $p 53$ suppresses the growth of cervical cancer in vitro and in vivo. Cancer Lett, 335, 242-50.

Liu G, Xia T, Chen X (2003). The activation domains, the proline-rich domain, and the $\mathrm{C}$-terminal basic domain in $p 53$ are necessary for acetylation of histones on the proximal p21 promoter and interaction with $\mathrm{p} 300 / \mathrm{CREB}-$ binding protein. J Biol Chem, 278, 17557-65.

Menendez D, Inga A, Resnick MA (2010). Potentiating the $p 53$ network. Discov Med, 10, 94-100.

Nunobiki O, Ueda M, Toji E, et al (2011). Genetic polymorphism of cancer susceptibility genes and HPV infection in cervical carcinogenesis. SAGE-Hindawi Access Res Pathol Res Int.

Scheffner M, Werness BA, Huibregtse JM, et al (1990). The E6 oncoprotein encoded by human papillomavirus types 16 and 18 promotes the degradation of $p 53$. Cell, 63, 1129-36.

Storey A, Thomas M, Kalita A, et al (1998). Role of a p53 polymorphism in the development of human papillomavirusassociated cancer. Nature, 393, 229-34

Tan S, Hougardy BMT, Meersma GJ, et al (2012). Human papilloma virus $16 \mathrm{E} 6 \mathrm{RNA}$ interference enhances cisplatin and death receptor-mediated apoptosis in human cervical carcinoma cells. Mol Parmacol, 81, 701-9.

Trigiante G, Lu X(2006). ASPPs and cancer. Nat Rev, 6, 217-26.

Zhi X, Honda K, Sumi T, et al (2007). Esyradiol-17beta regulates vascular endothelial growth factor and Bcl-2 expression in HHUA cells. Int J Oncol, 31, 1333-8 